\title{
Théologiques
}

Théologiques

\section{Le corps encore}

\section{Robert Jacques}

Volume 5, numéro 2, octobre 1997

Le corps : du dualisme à l'altérité

URI : https://id.erudit.org/iderudit/016653ar

DOI : https://doi.org/10.7202/016653ar

Aller au sommaire du numéro

Éditeur(s)

Faculté de théologie de l'Université de Montréal

ISSN

1188-7109 (imprimé)

1492-1413 (numérique)

Découvrir la revue

Citer ce document

Jacques, R. (1997). Le corps encore. Théologiques, 5(2), 3-8.

https://doi.org/10.7202/016653ar d'utilisation que vous pouvez consulter en ligne.

https://apropos.erudit.org/fr/usagers/politique-dutilisation/ 


\title{
Le corps encore
}

\author{
Robert JACQUES \\ Animateur de pastorale \\ Centre hospitalier Saint-Eustache
}

Les recherches sur la condition corporelle sont actuellement bien à la mode. On pourra consulter plus loin une brève bibliographie de parutions récentes. Les disciplines qui s'y intéressent sont multiples: anthropologie, philosophie, sociologie, neurosciences, éthique. En publiant un numéro sur cette thématique, Théologiques cède-t-elle au goût du jour, à la sensibilité du moment? Ne s'expose-t-elle pas ainsi au reproche d'accorder son attention à l'éphémère, comme il en va de toutes les modes? Ce qu'on lira ici, est-ce un autre vêtement jeté sur la nudité du corps? Jeté pour le protéger, le cacher ou le révéler? Le lecteur en décidera.

La phénoménologie élaborée à la suite de Husserl a consacré l'expression corps propre. Ce qualificatif donné au corps évoque tout à la fois l'unicité, la particularité et aussi la propriété. Et la propriété renvoie à un sujet qui dit mon corps. Certains s'empresseront de rappeler que la même phénoménologie a souvent déclaré : je n'ai pas un corps, mais je suis mon corps. Toutefois il faut être aveugle pour ne pas voir que l'adjectif possessif demeure dans cette affirmation. De quel ordre est cette possession, cette appropriation? Car il en va ici d'une subjectivité qui, malgré toutes les précautions anti-dualistes, continue de dire mon corps.

Cette question poursuit l'humain depuis longtemps. Elle était posée par ceux qui prônaient la fuite hors du corps ou sa maîtrise par la raison. $\mathrm{Ou}$ par Descartes qui, à la fois, refusait catégoriquement d'être « cet assemblage d'organes » et situait dans la glande pinéale le point de jonction entre le corps et sa raison. Aujourd'hui, les neurosciences et les sciences cognitives élaborent diverses hypothèses sur la production de la subjectivité ou de la conscience de soi : sécrétion du cerveau, résultante d'une sélection de groupes neuronaux, stratégie élaborée par l'appareil cérébral pour la survie de l'organisme, etc.. Ces sciences posent autrement mais crûment 
la question de la possession : Comment la subjectivité est-elle (liée à) un corps, (à) un objet physique, matériel? Les énonciateurs de cette interrogation se veulent radicalement monistes matérialistes : fini le vieux dualisme des philosophes et des religions. La description neuronale complète de la subjectivité (promise prochainement) annonce la fin de l'âme, du spirituel, etc. Ainsi pourra-t-on bientôt parler de la condition corporelle sans autre, qu'on appelle cet autre âme, raison, esprit, culture, Dieu. Mais cette ambition moniste trouve des détracteurs : ceux-ci y voient une réduction violente de l'être humain à un système biochimique aussi prodigieux soit-il; le discours inflationniste sur l'âme dans les nouvelles spiritualités témoigne de cette réaction.

Entre le triomphalisme de ceux-ci et le nihilisme des autres, une pensée de l'entre-deux est possible. Dans cet espace souvent étroit, sont offertes à la fois une critique de certaines appropriations culturelles du corps et la découverte de son insaisissable. Corps insaisissable? N'est-ce pas ce qu'on a longtemps dit de l'âme ou de l'esprit? Cet intangible corporel est peut-être le terreau de la liberté de l'humain et de sa confiance également. Ici, l'humain corporel rencontre véritablement un autre: son semblable lui aussi à jamais non maîtrisable. Les deux premières contributions données ici, avec un matériau souvent philosophique, dessinent à ce propos quelques traits.

Interrogeant divers éléments textuels chrétiens, les articles suivants mentionnent avec justesse les impasses d'une apologie de l'âme dans une réflexion sur la corporéité en christianisme. Leurs auteurs explorent la densité "charnelle" de cette tradition de foi à travers les thèmes pauliniens du "corps pour le Seigneur ", du "corps spirituel », ou encore les récits évangéliques de l'incarnation, de la transfiguration, de la cène, etc. Ils soulignent surtout que l'autre du corps est l'autre corps énonciateur de la parole. Des pistes sont ainsi dégagées vers l'intersubjectivité corporelle et l'expérience de Dieu à même la chair de l'humain.

Le corps est aussi une source étonnante de symbolique. Déjà, les développements au sujet d'une église corps $d u$ Christ l'illustrent. Certains courants de spiritualité chrétienne relativement récents $y$ ont puisé abondamment. La dernière contribution le rappelle à travers l'examen des motifs du Corps mystique et du Sacré-Cœur. Malgré leur aspect suranné, ces notions témoignent d'une métaphorisation souvent crue de la chair pour dire Dieu.

On notera des silences malheureux. Il y aurait encore à penser le corps féminin méprisé par les discours ou la violence, les chairs torturées par les idéologies et les totalitarismes d'hier et d'aujourd'hui, les questions éthiques liées aux mises en scène du corps dans les sciences, les tech- 
niques, etc. Aura-t-on échappé un peu au dualisme plusieurs fois critiqué? De ceci aussi, le lecteur est invité à décider.

\section{Suggestions de lecture}

Le question de l'expérience corporelle suscite actuellement une abondante publication d'ouvrages dans divers domaines de recherche. On trouvera ici plusieurs titres souvent très récents réunis sous quelques rubriques permettant une consultation plus facile. On s'étonnera probablement de ne pas trouver une section intitulée Psychologie et psychanalyse; les travaux en ces sciences humaines sont trop nombreux pour être ici indiqués; les personnes intéressées profiteront de l'impressionnante bibliographie sur la question rassemblée par B. Andrieu dans Le corps dispersé (voir ci-dessous). Pour quelques ouvrages, nous avons donné un rapide commentaire.

Anthropologie, ethnologie, sociologie

BAUD, Jean-Pierre. 1993. L'affaire de la main volée : une histoire juridique du corps. Paris, Seuil, 244 p.

BAUDRY, Patrick. 1991. Le corps extrême: approche sociologique des conduites à risque (Nouvelles études anthropologiques). Paris, L'Harmattan, $240 \mathrm{p}$.

EN COLLAB. 1994. "Les conduites extrêmes", Frontières, vol. 6, n 3. Intéressante introduction sur la mise en jeu du corps dans les activités sportives à risque.

GARNIER, Catherine (dir.). 1991. Le corps rassemblé : pour une perspective interdisciplinaire et culturelle de la corporéité. Montréal, Université du Québec, Ed. Agence d'Arc, 328 p.

HAINARD, Jacques et Roland KAEHR (éd.). 1983. Le corps enjeu. Neuchâtel, Musée d'ethnographie, 178 p.

VON KAENEL, Jean-Marie (dir.). Souffrances. Corps et âme, épreuves partagées (série Mutations, 142). Paris, Éditions Autrement, 217 p.

Réunion de textes souvent remarquables sur les expériences de la douleur et de la souffrance et soulignant à chaque fois comment le corps est engagé en celles-ci.

LE BRETON, David. 1992. Anthropologie du corps et modernité (Sociologie d'aujourd'hui). Paris, PUF, $2^{\mathrm{e}}$ éd. corrigée, $263 \mathrm{p}$.

L'auteur étudie les logiques sociales et culturelles au sein des pratiques, des discours et de l'imaginaire du corps actuellement. Son analyse met en évidence un dualisme persistant jusque dans les sciences et technologies médicales. 
LE BRETON, David. 1992. La sociologie du corps (Que sais-je?, 2678). Paris, PUF, $126 \mathrm{p}$.

PLESSNER, Helmuth. 1995. Le rire et le pleurer. Une étude des limites du comportement humain. Paris, Éditions de la Maison des sciences de l'homme, $215 \mathrm{p}$.

Cette "anthropologie philosophique , publiée en allemand en 1941 et traduite en français que récemment, explore la condition corporelle à partir de ces phénomènes que sont le rire et le pleurer en lesquels l'être humain est véritablement son corps.

SFEZ, Lucien. 1995. La santé parfaite. Critique d'une nouvelle utopie (L'histoire immédiate). Paris, Seuil, 398 p.

TARDAN-MASQUELIER, Ysé. 1996. Les chemins du corps (L'être et le corps). Paris, Albin Michel, $247 \mathrm{p}$.

Composé de textes donnés aux XIX ${ }^{e}$ Assises nationales du Yoga (Aix-les-Bains en 1995), ce recueil est représentatif de la diversité des discours tenus actuellement sur le corps; on y trouve un très intéressant article sur le sens du corps en christianisme de Henri Bourgeois.

VIGARELlO, Georges (dir.). Le gouvernement du corps (Communication, 56). Paris, Seuil, $250 \mathrm{p}$.

Éthique, philosophie et théologie

EN COLlAB. 1995. "Le corps : sujet ou objet? ", Cahiers de l'école des sciences philosophiques et religieuses 17-18.

ABEL, Olivier. 1995. "Le sujet à l'image d'un corps. Ni instrument, ni idole », Diogène 172, pp. 59-75.

Intéressant point de vue théologique d'un protestant sur la question du corps.

ANDRIEU, Bernard. 1993. Le corps dispersé : histoire du corps au XXe siècle (Santé, société et cultures). Paris, L'Harmattan, 451 p.

Ouvrage important présentant, après une brève "histoire du corps en Occident ", les approches psychanalytique, phénoménologique et neuroscientifique de la réalité corporelle.

ANDRIEU, Bernard. 1994. Les cultes du corps. Ethique et sciences (Santé, société et cultures). Paris, L'Harmattan, 299 p.

BARBARAS, Renaud. 1994. La perception. Essai sur le sensible (Optiques philosophie). Paris, Hatier, 79 p.

Bernard, Michel. 1995. Le corps (Points Essais, 317). Paris, Seuil (1976), $163 \mathrm{p}$.

GISEL, Pierre. 1992. Corps et esprit. Les mystères de l'incarnation et de la résurrection (Entrée libre, 23). Genève, Labor et Fides, 94 p. 
HALPÉRIN, Jean et Nicolas WEILL (dir.). 1996. Le Corps. Données et débats. Actes du XXXVe Colloque des intellectuels juifs de langue française. Paris, Albin Michel, $254 \mathrm{p}$.

HAROCHE, Michel-Pierre. 1990. L'âme et le corps. Philosophie et psychiatrie. Paris, Plon, $214 \mathrm{p}$.

HENRY, Michel. 1987. Philosophie et phénoménologie du corps: essai sur l'ontologie biranienne. Paris, $2^{\mathrm{e}}$ éd., PUF, $320 \mathrm{p}$.

IDE, Pascal. 1996. Le corps à cœur. Essai sur le corps humain (Enjeux). Paris, Saint-Paul, $378 \mathrm{p}$.

JOUBERT, Jacques. 1991. Le corps sauvé (Cogitatio fidei, 161). Paris, Cerf, $214 \mathrm{p}$.

L'auteur analyse quelques paradigmes en lesquels le christianisme a explicité sa compréhension de la condition corporelle et du salut (virginité, incarnation, eucharistie, corps mystique, etc.). Intéressant sans être essentiel.

KÜHN, Rolf. 1988. "Le corps retrouvé. Une phénoménologie subjective radicale appliquée à une investigation sur la corporéité ». Revue des Sciences philosophiques et théologiques 72, pp. 557-568.

LedURE, Yves. 1989. Transcendances. Essai sur Dieu et le corps. Paris, Desclée de Brouwer, $187 \mathrm{p}$.

MERLEAU-PONTY, Maurice. 1990. Phénoménologie de la perception (Tel, 4). Paris, Gallimard, $531 \mathrm{p}$.

MÜLlER, Denis et Carlo FOPPA (dir.). 1995. Le corps humain entre savoirs et pratiques: enjeux et éthiques et médicaux. Genève, Les Cahiers Médicaux-sociaux, vol. 39, $\mathrm{n}^{\mathrm{O}} 1,125 \mathrm{p}$.

RICHIR, Marc. 1993. Le corps. Essai sur l'intériorité (Optiques philosophie). Paris, Hatier, $77 \mathrm{p}$.

Excellente introduction, malgré sa brièveté, à la pensée philosophique du corps. L'auteur propose une vision de l'esprit en tant qu' « excès " de la condition corporelle.

Neurosciences, sciences cognitives, génétique, etc.

BERMÙDEZ, José Luis, Anthony MARCEL, Naomi EILAN (eds.). 1995. The Body and the Self. Cambridge (Mass), London (Eng), A Bradford Book - The MIT Press, $376 \mathrm{p}$.

Recueil de textes écrits par des philosophes et des scientifiques sur les thèmes $d u$ corps, de la conscience, du sens de la " propriété " du corps, du soi.

BERNARD, Jean et Marcel BESSIS (dir.). 1990. Soi et non-soi. Paris, Seuil, $311 \mathrm{p}$. 
Dialogues fascinants entre scientifiques et penseurs sur la reconnaissance de soi dans les organismes vivants et sur la subjectivité.

CRICK, Francis. 1994. L'hypothèse stupéfiante. À la recherche scientifique de l'âme. Paris, Plon, 424 p.

DAMASIO, Antonio R. 1995. L'erreur de Descartes. La raison des émotions. Paris, Éditions Odile Jacob, 368 p.

Ouvrage novateur par sa réintroduction de l'expérience corporelle dans les recherches sur le cerveau et l'esprit.

EDElman, Gerald M. 1994. Biologie de la conscience (Points). Paris, Seuil, $426 \mathrm{p}$.

GANASCIA, Jean-Gabriel. 1996. Les sciences cognitives (Dominos). Paris, Flammarion, $127 \mathrm{p}$.

MISSA, Jean-Noël. 1993. L'esprit-cerveau. La philosophie de l'esprit à la lumière des neurosciences (Pour demain). Paris, J. Vrin, $260 \mathrm{p}$.

Excellente vulgarisation en langue française des connaissances actuelles sur certains phénomènes cérébraux ("split-brain *, héminégligence, etc.) et la définition traditionnelle de la conscience.

SEARLE, John R. 1995. La redécouverte de l'esprit (nrf essais). Paris, Gallimard, $353 \mathrm{p}$.

SIMON, Michel (dir.), La peau de l'ame. Intelligence artificielle et neurosciences : approches pluridisciplinaires. Paris, Cerf, 1994, 443 p.

Impressionnant travail de vulgarisation des recherches actuelles sur les neurosciences, le psychisme (entre biologie et psychologie), sur l'intelligence artificielle et proposition d'une première réflexion théologique sur les questions issues de ces champs de recherche en regard de l'expérience chrétienne.

TIBON-CORNILLOT, Michel. 1992. Les corps transfigurés. Mécanisation du vivant et imaginaire biologique (Sciences ouvertes). Paris, Seuil, $311 \mathrm{p}$.

Recherche très intéressante sur les déplacements actuels dans la compréhension scientifique de la condition corporelle: l'auteur souligne le retour d'une finalité dans les recherches génétiques contredisant ainsi les principes mêmes d'une science "objective ".

VARELA, Francisco, Evan THOMPSON, Eleanor ROSCH. 1993. L'inscription corporelle de l'esprit. Sciences cognitives et expérience humaine (La couleur des idées). Paris, Seuil, 377 p.

Essai étonnant d'une mise en relation des sciences cognitives explorant nos modes de connaissance et la méditation bouddhique. 